\title{
DYNAMIC THEORY OF SUSPENSIONS WITH BROWNIAN EFFECTS*
}

\author{
R. CAFLISCH $\dagger$ AND G. C. PAPANICOLAOU $\ddagger$
}

Dedicated to J. B. Keller on his sixtieth birthday

\begin{abstract}
We consider a suspension of particles in a fluid settling under the influence of gravity and dispersing by Brownian motion. A mathematical description is provided by the Stokes equations and a Fokker-Planck equation for the one-particle phase space density. This is a nonlinear system that depends on a number of parametric functions of the spatial concentration of the particles. These functions are known only empirically or for dilute suspensions. We analyze the system, its stability, its asymptotic behavior under different scalings and its validity from more microscopic description. We summarize our conclusions at the end.
\end{abstract}

1. Introduction. The theory for the steady flow of a viscous fluid containing a suspension of solid particles is well developed. The recent work of Batchelor [2] and Hinch [16] presents an analysis which is correct asymptotically for small concentration of randomly distributed particles. They obtained expressions for the effective fluid stress and the gravity induced sedimentation speed, with their dependence on the particle concentration. A review of their work and additional references are found in Batchelor [3].

A number of studies of dynamic phenomena in suspensions have been made but the theory is far from complete. For the very simple problem of sedimentation through a stationary fluid, Crowley [10], [11], [12] has shown experimentally and analytically that a periodic array of spheres is unstable. Also, Siano [25] has demonstrated experimentally that a spatially uniform distribution of sedimenting particles is unstable for some range of parameters. Cohen and Hagan [9] have discussed Siano's results using a free energy function. Other studies of stability in similar systems are found in [13], [15], [17]. A general reference for the static and dynamic theories of suspensions is Happel and Brenner [14].

In this paper we present a dynamic theory for a suspension of particles subject to gravity and Brownian motion. We demonstrate the possibility of linear instability for a random, homogeneous distribution of particles and find a description of the resulting nonlinear equilibrium. This instability depends crucially on how the strength of the Brownian diffusion varies with the particle concentration. Although we are unable to find this diffusion coefficient exactly, we analyze some related problems that involve interaction effects for particles under Brownian motion. From this analysis one can get some idea about the properties of the diffusion coefficient that are relevant in the rest of the paper.

The configuration of particles is described by a distribution function $f(x, \xi, t)$ for the particle position $x$ and velocity $\xi$ at time $t$. It satisfies a Fokker-Planck equation in which the coefficients depend on the local volume fraction $\phi=\phi(x, t)$ of particles. The equation includes the effects of gravity, drag by the fluid, and diffusion in velocity space. After it and the effective fluid equation are formulated in $\S 2$, the equations are nondimensionalized in $\S 3$, and various scalings are discussed.

* Received by the editors August 9, 1982.

$\dagger$ Department of Mathematics, Stanford University, Stanford, California 94305. The research of this author was supported by the National Science Foundation, the Army Research Office, the Air Force Office of Scientific Research and the Office of Naval Research.

‡Courant Institute of Mathematical Sciences, New York University, New York, New York 10012. The research of this author was supported by the Office of Naval Research under grant N00014-81-K-0002. 
In $\S 4$ we linearize the equation about a spatially uniform state and derive the corresponding dispersion relation. This dispersion relation is analyzed in $\S 5$ to show the possibility of instability for some finite interval of wavenumber $k$.

Since the linearized equations may contain instability, a nonlinear theory is needed. The nonlinear Kramers-Smoluchovski limit [20] of weak spatial dependence (or strong velocity diffusion) is performed in $\S 6$, using an analogue of the ChapmanEnskog expansion [8]. In that limit we derive a nonlinear equation for the local volume fraction $\phi(x, t)$. This continuum theory for the suspension is the nonlinear analogue of the preceding linear analysis. Steady solutions of this equation are nonlinear equilibrium states. In one space dimension such solutions are discussed in $\S 7$ and in more detail, along with some results on their stability, in [21].

In $\S 8$ we consider the form of the $\mathrm{N}$-particle linear Kramers-Smoluchovski limit which is an $N$-particle interacting diffusion process. In $\S 9$ we discuss the existence and dependence on concentration of the self-diffusion coefficient for interacting Brownian motions. At the end of this section we argue heuristically that the velocity diffusion coefficient used in $\S \S 2$ to 7 behaves more like the self-diffusion coefficient than the bulk diffusion coefficient for a system of interacting Brownian motions. The former decreases with concentration while the latter increases in general [4].

2. Formulation of the equations. We shall adopt a mathematical description of the suspension based on the Stokes equation for the fluid and a Fokker-Planck equation for the one-particle phase space distribution function. This description is compatible with the two-fluid modelling of the suspension [13], [15] but contains more information allowing for the conclusions we wish to draw. In Appendix A we show that the usual two-fluid description, including Brownian effects, is inadequate for our purposes here.

Let $v_{p}(t, x)$ and $v_{f}(t, x)$ be the average velocities of the particle and fluid phase that have densities $\rho_{p}$ and $\rho_{f}$ respectively. Let $\phi(t, x)$ be the volume fraction density of the particles. The two-fluid continuum equations for the suspension have the following form [13], [15]:

$$
\begin{aligned}
& \phi_{t}+\nabla \cdot\left(\phi v_{p}\right)=0 \\
& (1-\phi)_{t}+\nabla \cdot\left((1-\phi) v_{f}\right)=0 \\
& \rho_{p} \phi\left(v_{p t}+\left(v_{p} \cdot \nabla\right) v_{p}-g\right)=-\phi \nabla p-\phi S\left(v_{p}-v_{f}\right) \\
& \rho_{f}(1-\phi)\left(v_{f t}+\left(v_{f} \cdot \nabla\right) v_{f}-g\right)=-(1-\phi) \nabla p+\phi S\left(v_{p}-v_{f}\right)+(1-\phi) \mu \Delta v_{f} .
\end{aligned}
$$

In (2.1)-(2.4) $g$ denotes the acceleration of gravity vector pointing downward, $p$ denotes the pressure, $\mu$ the viscosity of the fluid, and $S$ the coefficient of interaction for the force exerted by one fluid on the other. At low concentration, $\phi$,

$$
S=\frac{(1-\phi)^{2}}{1-6.55 \phi} \frac{9}{2} \frac{\mu}{a^{2}}
$$

where $a$ is the radius of the particles. This is obtained as follows. Equations (2.1)-(2.4) have the equilibrium solution

$$
\begin{aligned}
& v_{p}-v_{f}=\frac{1-\phi}{S} g\left(\rho_{p}-\rho_{f}\right), \\
& p=\left(\phi \rho_{1}+(1-\phi) \rho_{2}\right) g \cdot x,
\end{aligned}
$$

where $v_{p}$ and $v_{f}$ are constant now. The solution (2.6)-(2.7) corresponds to uniform 
settling in the presence of gravity. When the average velocity

$$
u=\phi v_{p}+(1-\phi) v_{f}
$$

equals zero, we may identify $v_{p}$ with the settling velocity and for low concentration $\phi$, Batchelor [2] has calculated the dependence of the settling velocity on $\phi$ to obtain

$$
v_{p}=(1-6.55 \phi) v_{\mathrm{sT}}
$$

where $v_{\mathrm{ST}}$ is the Stokes settling velocity of a free particle,

$$
v_{\mathrm{ST}}=\frac{g 4 \pi a^{3}\left(\rho_{p}-\rho_{f}\right) / 3}{6 \pi \mu a} .
$$

Thus, from $\phi v_{p}+(1-\phi) v_{f}=0,(2.6),(2.9)$ and (2.10), we can solve for $S$ in (2.6) to obtain the expression (2.5).

With the determination (2.5) for $S$, equations (2.1)-(2.4) are the usual two-fluid equations for the suspension. If we add a term of the form $\phi \sigma \nabla \phi$ on the right side of (2.3) the new system includes Brownian effects and is analyzed further in Appendix A. As we indicated above we shall not use (2.1)-(2.4) but we shall replace (2.1) and (2.3) by a Fokker-Planck equation. Our system of equations will contain (2.2), (2.4) and the Fokker-Planck equation

$$
f_{t}+\xi \cdot \nabla f+\nabla_{\xi} \cdot\left[\left(g \gamma\left(1-\frac{\rho_{f}}{\rho_{p}}\right)-M^{-1} 6 \pi \mu a \theta(\xi-u)\right) f\right]=D \delta \nabla_{\xi}^{2} f .
$$

Here $f=f(t, x, \xi)$ is the phase space volume fraction density of particles, i.e. the average volume of particles at $x$ (per unit volume) going with velocity $\xi$ (per unit volume in velocity space). The average volume fraction $\phi$, or concentration of particles, is the $\xi$-integral of $f$

$$
\phi=\int f d \xi
$$

while the average particle velocity $v_{p}$ is the $\xi$-integral of $\xi f$

$$
\phi v_{p}=\int \xi f d \xi .
$$

In (2.11) we introduced some additional notation as follows. The mass of a particle is

$$
M=\frac{4}{3} \pi a^{3} \rho_{p},
$$

while $\mu$ is the fluid viscosity and $g$ the acceleration of gravity vector as before. The velocity diffusivity

$$
D=\frac{k T}{M} \frac{6 \pi \mu a}{M},
$$

which is the Einstein formula with $k=$ Boltzmann's constant and $T=$ the temperature of the suspension, assumed constant. The functions $\gamma(\phi), \theta(\phi)$ and $\delta(\phi)$ are dimensionless and

$$
\gamma(\phi)=1-\phi,
$$

modifies the gravitational acceleration to account for the additional buoyancy of the fluid due to the suspended particles. The function $\theta(\phi)$ gives the modification of the Stokes drag on a particle due to the presence of the other particles. Notice 
that the drag term $M^{-1} 6 \pi \mu a \theta(\xi-u)$ in $(2.11)$ is computed with respect to the velocity of the particle $\xi$ relative to the volume average velocity of the suspension $u$ given by (2.8). From (2.11) the settling velocity of a particle at equilibrium is obtained by the balance of drag and gravitational force and equals $v_{\mathrm{ST}} \gamma / \theta$ where $v_{\mathrm{ST}}$ is given by (2.10). As in $(2.9)$ we may equate this to $(1-6.55 \phi) v_{\mathrm{ST}}$ according to Batchelor's formula [4], where $\phi$ is small. This gives

$$
\theta(\phi)=\frac{1-\phi}{1-6.55 \phi}
$$

It should be remarked that there are also empirical and experimental investigations that lead to a determination of the settling velocity as function of concentration (which in turn determines $\theta(\phi))$. In general

$$
\frac{\gamma}{\theta}=\left(1-\tilde{c}_{1} \phi^{1 / 3}\right)\left(1-\tilde{c}_{2} \phi\right),
$$

for small $\phi$ where $\tilde{c}_{1}$ and $\tilde{c}_{2}$ are constants. For very small particles [2], [3], [6], [16] $\tilde{c}_{1}=0, \tilde{c}_{2}=6.55$ as in Batchelor's theoretical calculation. For larger particles [1], [22] $\tilde{c}_{1}=.75$ and $\tilde{c}_{2}=2.15$.

The function $\delta(\phi)$ defined in (2.11) is difficult to determine even at low concentrations and there seem to be no experimental data that help in estimating it in a reasonably direct way. By analogy with a simpler problem, we shall argue in $\S \S 8$ and 9 that $\delta(\phi)$ decreases rapidly with increasing $\phi$. This means that the velocity diffusivity, equal to one when $\phi=0$, decreases rapidly with increasing concentration.

We collect now the equations we shall use to model the suspension.

$$
\begin{array}{rr}
(1-\phi)_{t}+\nabla \cdot\left(v_{f}(1-\phi)\right)=0, & \text { continuity equation for fluid, } \\
\rho_{f}(1-\phi)\left(v_{f t}+\left(v_{f} \cdot \nabla\right) v_{f}-g\right)=-(1-\phi) \nabla p+\phi S\left(v_{p}-v_{f}\right)+(1-\phi) \mu \Delta v_{p}, & \text { momentum equation for fluid, } \\
f_{t}+\xi \cdot \nabla f+\nabla_{\xi} \cdot\left[\left(g \gamma\left(1-\frac{\rho_{f}}{\rho_{p}}\right)-M^{-1} 6 \pi \mu a \theta(\xi-u)\right) f\right]=D \delta \nabla_{\xi}^{2} f, \\
\text { Fokker-Planck equation for particles. }
\end{array}
$$

In these equations $\phi$ and $v_{p}$ are related to $f$ by (2.12) and (2.13), $S$ is given by (2.5), $M$ and $D$ are given by (2.14) and (2.15), $\gamma(\phi)$ and $\theta(\phi)$ are given by (2.16) and (2.17) while $\delta(\phi)$ is discussed following (2.18) and in subsequent sections. Note also that $u$ in (2.21) is the volume average velocity (2.8). System (2.19)-(2.21) is a complicated nonlinear system to be supplemented with initial and boundary conditions.

In modelling the suspension by (2.19)-(2.21) a number of physical mechanisms have been left out because they have negligible effects in the range of parameters which we have in mind. These include effects of particle rotation and inertia causing transverse particle motion, temperature variations, and boundary conditions. Additional complications could be caused by considering nonspherical particles with some size and shape distribution and by allowing for particle aggregation. Our focus is on only two mechanisms: gravitational settling and Brownian dispersion.

We shall now show that the Fokker-Planck equation (2.21) is compatible with the particle continuity and momentum equations (2.1) and (2.3) of the two-fluid model (2.1)-(2.4).

Integrating (2.21) with respect to $\xi$ and using (2.12) and (2.13) yields immediately the continuity equation (2.1). Multiplying (2.21) by $\xi$, integrating with respect to $\xi$ 
and using (2.12) and (2.13) yields

$$
\left(\phi v_{p}\right)_{t}+\nabla \cdot \int \xi \xi f d \xi-g \gamma \frac{\left(\rho_{p}-\rho_{f}\right)}{\rho_{p}} \phi+\frac{6 \pi \mu a \theta}{\mu}\left(v_{p}-u\right) \phi=0
$$

We may write

$$
\nabla \cdot \int \xi \xi f d \xi=\nabla \cdot(\phi v v)+\nabla(\phi P)
$$

where $P$ plays the role of pressure and is given by

$$
\phi P=\int\left(\xi-v_{p}\right)^{2} f d \xi
$$

We have assumed that $P$ is isotropic (i.e. a scalar) since for the present discussion we may restrict ourselves to isotropic $f$. Using now the continuity equation (2.1) and $(2.23)$ in (2.22) we obtain

$$
\rho_{p} \phi\left(v_{p t}+\left(v_{p} \cdot \nabla\right) v_{p}\right)+\rho_{p} \nabla(\phi P)-g(1-\phi) \phi\left(\rho_{p}-\rho_{f}\right)=-S \phi\left(v_{p}-v_{f}\right) .
$$

To obtain the right-hand side in (2.24) we used (2.14), (2.17), (2.8) and the definition of $S$ (2.5). We can write (2.24) in the form (2.3) if we identify the pressure $p$ with an appropriate expression involving $P$ (which can be thought of as a function of $\phi$ as in the classical kinetic theory of gases) and the gravity term.

The above discussion clarifies the differences between the descriptions (2.1)-(2.4) and $(2.19)-(2.21)$ for the suspension. We shall use $(2.19)-(2.21)$ because we want more refined Brownian effects. As a final remark regarding (2.19)-(2.21) we note that we always have

$$
\nabla \cdot u=0
$$

where $u=\phi v_{p}+(1-\phi) v_{f}$ appears in (2.21). This follows immediately from (2.1) and (2.19) and of course (2.1) is always a consequence of (2.21).

3. Nondimensionalization and scalings. We shall first nondimensionalize the equations (2.19)-(2.21), then discuss possible scalings. Three relevant parameters are the Stokes settling time, the Stokes velocity (cf. (2.10)) of a settling particle, and the root mean square of the velocity fluctuations:

$$
\begin{aligned}
& \tau_{\mathrm{ST}}=M / 6 \pi \mu a, \\
& v_{\mathrm{ST}}=g\left(1-\rho_{f} / \rho_{p}\right) \tau_{\mathrm{ST}}=\left|v_{\mathrm{ST}}\right| \hat{z}, \\
& \left(D \tau_{\mathrm{ST}}\right)^{1 / 2}=(k T / M)^{1 / 2},
\end{aligned}
$$

in which $\hat{z}$ is the unit vector pointing downward.

Let $\tau$ and $X$ be typical time and length scales. We nondimensionalize (2.19)-(2.21) by choosing new independent variables $t^{\prime}, x^{\prime}, \xi^{\prime}$ defined by

$$
\xi=\left(D \tau_{\mathrm{ST}}\right)^{1 / 2} \xi^{\prime}, \quad t=\tau t^{\prime}, \quad x=X x^{\prime} .
$$

We also define $v^{\prime}, u^{\prime}$, and $p^{\prime}$ by

$$
\begin{array}{ll}
v_{p}=\left(D \tau_{\mathrm{ST}}\right)^{1 / 2} v_{p}^{\prime}, & v_{f}=\left(D \tau_{\mathrm{ST}}\right)^{1 / 2} v_{f}^{\prime}, \\
u=\left(D \tau_{\mathrm{ST}}\right)^{1 / 2} u^{\prime}, & p=\frac{\rho_{f} X\left(D \tau_{\mathrm{ST}}\right)^{1 / 2}}{\tau} p^{\prime} .
\end{array}
$$


Four relevant dimensionless parameters are

$$
\begin{array}{ll}
A=\frac{-\tau\left|v_{\mathrm{ST}}\right|}{\tau_{\mathrm{ST}}\left(D \tau_{\mathrm{ST}}\right)^{1 / 2}}, & E=\frac{2 \tau a^{2}}{9 \tau_{\mathrm{ST}} X^{2}} \frac{\rho_{p}}{\rho_{f}} \\
B=\tau\left(D \tau_{\mathrm{ST}}\right)^{1 / 2} / X, & F=\left(\frac{X}{a}\right)^{2} E . \\
C=\tau / \tau_{\mathrm{ST}}, &
\end{array}
$$

Substituting (3.4)-(3.6) into (2.19)-(2.21) and dropping prime yields the system ${ }^{1}$

$$
\begin{aligned}
& (1-\phi)_{t}+B \nabla \cdot\left(v_{f}(1-\phi)\right)=0, \\
& v_{f t}=-\nabla p+\phi(1-5.55 \phi) F\left(v_{p}-v_{f}\right)+E \Delta v_{f}, \\
& f_{t}+B \xi \cdot \nabla f+\nabla_{\xi} \cdot[(A \gamma \hat{z}-C \theta(\xi-u)) f]=C \delta \nabla_{\xi}^{2} f .
\end{aligned}
$$

In these equations $A, B, C, E$ and $F$ are constants depending on the physical parameters, while $\gamma, \theta$, and $\delta$ are functions of the particle volume fraction $\phi$ and $\hat{z}$ is a unit vector in the $z$ direction.

We shall study the time and space variations and the stability of a suspension which is nearly uniform. Part of our motivation is a very interesting set of experiments by Siano [25] in which a spatially uniform suspension was found to be unstable for some range of parameters. We shall describe the scaling corresponding to those experiments as well as the scaling for linearization around a uniform state. The resulting equations will be analyzed in $\$ \S 4-7$.

(i) Siano's experiments. Typical parameter values in his experiments are

$$
\begin{aligned}
& a=.5 \times 10^{-4} \mathrm{~cm}, \quad \rho_{f}=1.00 \mathrm{gm} \mathrm{cm}^{-3}, \\
& \mu=.01 \text { dynes sec } \mathrm{cm}^{-2}, \quad T=293 \mathrm{deg} \mathrm{K} \text {, } \\
& \rho_{p}=1.05 \mathrm{gm} \mathrm{cm}^{-3}, \quad \phi=.001 .
\end{aligned}
$$

With these values and the constants $k=1.38 \times 10^{-16} \mathrm{ergs} /{ }^{\circ} \mathrm{K}$ and $g=980 \mathrm{~cm} \mathrm{sec}^{-1}$, we obtain

$$
\begin{aligned}
& \tau_{\mathrm{ST}}=.6 \times 10^{-7} \mathrm{sec}, \\
& v_{\mathrm{ST}}=.3 \times 10^{-6} \mathrm{~cm} \mathrm{sec}^{-1}, \\
& \left(D \tau_{\mathrm{ST}}\right)^{1 / 2}=.3 \mathrm{~cm} \mathrm{sec}^{-1} .
\end{aligned}
$$

The observed patterns have space and time scales of cm's and hours, therefore we choose

$$
X=1 \mathrm{~cm}, \quad \tau=10^{5} \mathrm{sec}
$$

The resulting dimensionless parameters are

$$
\begin{array}{ll}
A=-1.6 \times 10^{7}, & E=.9 \times 10^{4}, \\
B=.3 \times 10^{5}, & F=3.6 \times 10^{12} . \\
C=1.6 \times 10^{12}, &
\end{array}
$$

\footnotetext{
${ }^{1}$ From here on we shall neglect the inertial term $\left(v_{f} \cdot \nabla\right) v_{f}$ in $(2.20)$. In addition, the gravitational term is absorbed in the pressure term.
} 
Therefore we let ${ }^{2}$

and (3.9) becomes

$$
A=\frac{\alpha}{\varepsilon}, \quad B=\frac{1}{\varepsilon}, \quad C=\frac{c}{\varepsilon^{2}}, \quad E=\frac{e}{\varepsilon}
$$

$$
\varepsilon f_{t}+\xi \cdot \nabla f+\nabla_{\xi} \cdot\left(\alpha \gamma \hat{z}-\frac{c}{\varepsilon} \theta(\xi-u)\right) f=\frac{c}{\varepsilon} \delta \nabla_{\xi}^{2} f
$$

(ii) Small perturbations of a uniform state. As will be shown in $\S 4$, there is a spatially homogeneous solution $f_{0}(\xi)$ of (3.15). We look for a solution of the form

$$
f(t, x, \xi)=f_{0}(\xi)+\bar{\varepsilon} f_{1}(t, x, \xi)+O\left(\bar{\varepsilon}^{2}\right)
$$

in which $\bar{\varepsilon}$ is small. The perturbation $f_{1}$ will satisfy the linearization of (3.16) about $f=f_{0}$. The resulting equation is valid for any values of the parameters $A, B, C$. We do not make use of the ordering (3.14) in $\S 4$.

Note. To simplify the presentation and the algebra, we shall often consider the case $\alpha=0$ in which gravitational effects are excluded. This occurs if the particles are neutrally buoyant.

The nonlinear analysis of $\S \S 6$ and 7 for equation (3.15) which comes from the scaling (3.14) could also be performed for other scalings, such as $A=\alpha / \varepsilon^{2}, B=O(1)$, $C=c / \varepsilon^{2}$. The analysis is more complicated than that of $\S \S 6$ and 7 , since the gravitational terms come in at the lowest order.

4. Linearization about the homogeneous state. There is a solution of the FokkerPlanck equation (3.9) with $u=0^{3}$ which is spatially uniform with a Gaussian distribution of particle velocities. This solution is

$$
f_{0}(\xi)=\phi_{0}(2 \pi \sigma)^{-3 / 2} \exp \left\{-\left(\xi-v_{0}\right)^{2} / 2 \sigma\right\},
$$

in which

$$
v_{0}=\hat{z} A \gamma / C \theta, \quad \sigma=\delta / \theta .
$$

The volume fraction $\phi_{0}$ is an arbitrary constant, $v_{0}$ is the sedimentation speed, and the velocity variance $\sigma$ is evaluated at $\phi_{0}$.

We look for a solution in the form (3.16) with

$$
\phi=\phi_{0}+\bar{\varepsilon} \phi_{1}+O\left(\bar{\varepsilon}^{2}\right) \text {. }
$$

The linearized equations for $f_{1}$ and $\phi_{1}$ are

$$
f_{1 t}=L^{*} f_{1}+h,
$$

in which

$$
\begin{aligned}
& L^{*} f_{1}=-\xi \cdot \frac{\partial}{\partial x} f_{1}-\nabla_{\xi} \cdot\left[(A \gamma \hat{z}-C \theta \xi) f_{1}\right]+C \delta \nabla_{\xi}^{2} f_{1}, \\
& h=-\nabla_{\xi} \cdot\left[\left(\hat{z} A \gamma^{\prime} \phi_{1}-\xi C \theta^{\prime} \phi_{1}\right) f_{0}\right]+C \delta^{\prime} \phi_{1} \nabla^{2} f_{0} \\
& \quad=\nabla_{\xi} \cdot\left(h_{1} f_{0}\right)+\nabla_{\xi} \cdot\left(\xi h_{2} f_{0}\right),
\end{aligned}
$$

\footnotetext{
${ }^{2}$ The ordering (3.14) is obviously not in complete agreement with (3.13). It merely helps construct approximations and is not used in a quantitative but in a qualitative manner. That is, we give no error estimates for the approximation.

${ }^{3}$ We shall analyze (3.9) with $u=0$ throughout this section except at the end where we comment on the case $u \neq 0$.
} 


$$
h_{1}=\left(-A \gamma^{\prime}+A \gamma \delta^{\prime} / \delta\right) \phi_{1} \hat{z}=A \gamma\left(\frac{\sigma^{\prime}}{\sigma}+\frac{\theta^{\prime}}{\theta}-\frac{\gamma^{\prime}}{\gamma}\right) \phi_{1} \hat{z}
$$

$$
\begin{aligned}
& h_{2}=\left(C \theta^{\prime}-C \theta \delta^{\prime} / \delta\right) \phi_{1}=-C \theta \frac{\sigma^{\prime}}{\sigma} \phi_{1}, \\
& \phi_{1}=\int f_{1} d \xi .
\end{aligned}
$$

In these equations we have denoted $\gamma=\gamma\left(\phi_{0}\right), \gamma^{\prime}=\gamma^{\prime}\left(\phi_{0}\right)$, etc.

For notational convenience we make the following rescaling (which depends on $\left.\phi_{0}\right)$ :

$$
\begin{array}{ll}
s=C \theta t, & y=C \theta \sigma^{-1 / 2} x, \\
\eta=\sigma^{-1 / 2} \xi, & w_{0}=\sigma^{-1 / 2} v_{0}, \\
g_{i}=\sigma^{3 / 2} f_{i}, & m=\left(\sigma^{3 / 2} / c \theta\right) h, \\
m_{1}=\left(\sigma^{1 / 2} c \theta\right)^{-1} h_{1}, & m_{2}=(c \theta)^{-1} h_{2} .
\end{array}
$$

Then (4.1) and (4.4)-(4.8) become

$$
\begin{aligned}
& g_{0}=\phi_{0}(2 \pi)^{-3 / 2} \exp \left\{-\frac{1}{2}\left(\eta-w_{0}\right)^{2}\right\}, \\
& \frac{\partial}{\partial s} g_{1}=K^{*} g_{1}+m, \\
& K^{*} g_{1}=-\eta \cdot \frac{\partial}{\partial y} g_{1}-\nabla_{\eta} \cdot\left[\left(w_{0}-\eta\right) g_{1}\right]+\nabla_{\eta}^{2} g_{1}, \\
& m=\nabla_{\eta} \cdot\left(m_{1} g_{0}\right)+\nabla_{\eta} \cdot\left(\eta m_{2} g_{0}\right), \\
& m_{1}=w_{0}\left(\frac{\sigma^{\prime}}{\sigma}+\frac{\theta^{\prime}}{\theta}-\frac{\gamma^{\prime}}{\gamma}\right) \phi_{1}, \\
& m_{2}=-\frac{\sigma^{\prime}}{\sigma} \phi_{1}, \\
& \phi_{1}=\int g_{1} d \eta .
\end{aligned}
$$

In looking for solutions of the homogeneous equations (4.10)-(4.15), we shall first consider $m$ as an inhomogeneity. To find the Green's function for $K^{*}$, we consider the stochastic process $(y(t), \eta(t))$ defined by

$$
\begin{array}{ll}
\dot{y}=\eta, & y(0)=y_{0}, \\
\dot{\eta}=w_{0}-\eta+\sqrt{2} \dot{\omega}, & \eta(0)=\eta_{0},
\end{array}
$$

with $\dot{\omega}$ the standard white noise. This process has infinitesimal generator $K$, the formal adjoint of $K^{*}$. Its solution is given by the following stochastic integrals:

$$
\begin{aligned}
& \eta(s)=m_{\eta}(s)+\sqrt{2} \int_{0}^{s} e^{-\left(s-s_{0}\right)} d \omega\left(s_{0}\right), \\
& y(s)=m_{y}(s)+\sqrt{2} \int_{0}^{s}\left(1-e^{-\left(s-s_{0}\right)}\right) d \omega\left(s_{0}\right),
\end{aligned}
$$


with mean velocity and position

$$
\begin{aligned}
& m_{\eta}(s)=w_{0}+e^{-s} \eta_{0}, \\
& m_{y}(s)=y_{0}+w_{0} s+\left(1-e^{-s}\right) \eta_{0} .
\end{aligned}
$$

This process is jointly Gaussian with covariances

$$
\begin{aligned}
& \Sigma_{22}(s)=\operatorname{var}(\eta)=2 \int_{0}^{s} e^{-2\left(s-s_{0}\right)} d s_{0}=\left(1-e^{-2\left(s-s_{0}\right)}\right), \\
& \Sigma_{11}(s)=\operatorname{var}(y)=2 \int_{0}^{s}\left(1-e^{-\left(s-s_{0}\right)}\right)^{2} d s_{0}
\end{aligned}
$$

$$
\begin{aligned}
& =2\left(s-\frac{3}{2}+\left(2 e^{-s}-\frac{1}{2} e^{-2 s}\right)\right), \\
\Sigma_{12}(s)=\operatorname{cov}(y, \eta) & =2 \int_{0}^{s} e^{-\left(s-s_{0}\right)}\left(1-e^{-\left(s-s_{0}\right)}\right) d s_{0} \\
& =\left(1-e^{-s}\right)^{2} .
\end{aligned}
$$

The resulting transition density $G\left(s, y, y_{0}, \eta, \eta_{0}\right)$, which is the Green's function for $K^{*}$, is

$$
G\left(s, y, y_{0}, \eta, \eta_{0}\right)
$$

$$
\begin{gathered}
=(2 \pi)^{-3} \Delta^{-3 / 2} \exp \left\{-\frac{1}{2 \Delta(s)}\left[\Sigma_{22}\left(y-m_{y}\right)^{2}\right.\right. \\
\left.\left.-2 \Sigma_{12}\left(y-m_{y}\right) \cdot\left(\eta-m_{\eta}\right)+\Sigma_{11}\left(\eta-m_{\eta}\right)^{2}\right]\right\}, \\
\Delta(s)=\Sigma_{11} \Sigma_{22}-\Sigma_{12}^{2} .
\end{gathered}
$$

The validity of this Green's function can be verified directly without the use of the stochastic equations (4.16), (4.17).

A useful marginal density function is

$$
G_{M}\left(s, y, y_{0}, \eta_{0}\right)=\int G d \eta=\left(2 \pi \Sigma_{11}\right)^{-3 / 2} \exp \left(-\left(y-m_{y}\right)^{2} / 2 \Sigma_{11}\right) .
$$

This is a function of $y-y_{0}$ since

$$
y-m_{y}=\left(y-y_{0}\right)-w_{0} s-\left(1-e^{-s}\right) \eta_{0} .
$$

Its Fourier transform in $\left(y-y_{0}\right)$ is

$$
\hat{G}_{M}\left(s, k, \eta_{0}\right)=\exp \left\{i k \cdot \eta_{0}\left(1-e^{-s}\right)+i k \cdot w_{0} s-\frac{1}{2} \Sigma_{11}(s) k^{2}\right\} .
$$

The solution $g_{1}$ of $(4.11)$ is given by

$$
g_{1}(s, y, \eta)=\int_{0}^{s} \int_{R^{3}} \int_{R^{3}} G\left(s-s_{0}, y, y_{0}, \eta, \eta_{0}\right) m\left(s_{0}, y_{0}, \eta_{0}\right) d \eta_{0} d y_{0} d s_{0}
$$

and its mass density is

$$
\begin{aligned}
\phi_{1}(s, y) & =\int g_{1} d \eta \\
& =\int_{0}^{s} \int_{R^{3}} \int_{R^{3}} G_{M}\left(s-s_{0}, y, y_{0}, r_{0}\right) m\left(s_{0}, y_{0}, \eta_{0}\right) d \eta_{0} d y_{0} d s_{0}
\end{aligned}
$$


In order to Fourier transform this, we first calculate for any $\alpha$,

$$
\begin{aligned}
\int_{R^{3}} e^{i k \cdot \eta_{0} \alpha} \hat{m}\left(s_{0}, k, \eta_{0}\right) d \eta_{0} \\
\quad=\phi_{0}\left\{-i \alpha k \cdot \hat{m}_{1}+\left(\alpha^{2} k^{2}-i \alpha k \cdot w_{0}\right) \hat{m}_{2}\right\} \exp \left(-\frac{1}{2} \alpha^{2} k^{2}+i \alpha k \cdot w_{0}\right) \\
\quad=\phi_{1}\left(d_{1} k^{2} \alpha^{2}-i d_{2} k \cdot w_{0} \alpha\right) \exp \left(-\frac{1}{2} \alpha^{2} k^{2}+i \alpha k \cdot w_{0}\right),
\end{aligned}
$$

in which

$$
d_{1}=-\frac{\sigma^{\prime}}{\sigma} \phi_{0}, \quad d_{2}=\left(\frac{\theta^{\prime}}{\theta}-\frac{\gamma^{\prime}}{\gamma}\right) \phi_{0} .
$$

Using (4.29) and (4.32) with $\alpha=\left(1-e^{-\left(s-s_{0}\right)}\right)$, we can transform (4.31) to obtain

$$
\begin{aligned}
\hat{\phi}_{1}(s, k)= & \int_{0}^{s} \Psi\left(s-s_{0}, k\right) \hat{\phi}_{1}\left(s_{0}, k\right) d s_{0} \\
\Psi(s, k)= & \left(d_{1} k^{2}\left(1-e^{-s}\right)^{2}-i d_{2} k \cdot w_{0}\left(1-e^{-s}\right)\right) \\
& \cdot \exp \left\{-\frac{1}{2}\left(1-e^{-s}\right)^{2} k^{2}+i\left(1-e^{-s}\right) k \cdot w_{0}+i k \cdot w_{0} s-\frac{1}{2} \Sigma_{11}(s) k^{2}\right\}
\end{aligned}
$$

After taking the Laplace transform in $s$ of (4.34) and dividing by $\tilde{\phi}_{1}(\lambda, k)$, the Laplace transform of $\hat{\phi}_{1}(s, k)$, we obtain the following dispersion relation:

$$
1=\tilde{\Psi}(\lambda, k) \equiv \int_{0}^{\infty} e^{-\lambda s} \Psi(s, k) d s .
$$

This is an implicit equation for $\lambda$ as a function of $k$.

If we had started from the system (3.7)-(3.9) with $u \neq 0$, there would be a term containing $u$ in $m_{1}$. Only $k \cdot \hat{m}_{1}$ appears in (4.32), and the corresponding term $k \cdot \hat{u}=(\nabla \cdot u) \hat{~ i s ~ z e r o ~ b e c a u s e ~ o f ~(2.25) . ~ T h e r e f o r e ~(4.32) ~ a n d ~ t h e ~ r e s u l t i n g ~ d i s p e r s i o n ~}$ relation (4.36) would be unchanged and the linearized analysis presented here is valid in general.

5. The dispersion relation. We shall examine the dispersion relation (4.36) in detail for $k$ small. For simplicity we shall at first exclude gravitational effects, as would occur for neutrally buoyant particles.

Neutrally buoyant particles. In the absence of gravitational effects, the sedimentation speed $w_{0}$ is zero. From (4.23) and (4.35) we then find

$$
\Psi(s, k)=d_{1} k^{2}\left(1-e^{-s}\right)^{2} \exp \left\{-k^{2}\left(-1+s+e^{-s}\right)\right\},
$$

$$
\begin{aligned}
\tilde{\Psi}(s, k) & =\int_{0}^{\infty} e^{-\lambda s} \Psi(s, k) d s \\
& =d_{1} k^{2} e^{k^{2}}\left[\left(\lambda+k^{2}\right)^{-1}+\int_{0}^{\infty} e^{-\left(\lambda+k^{2}\right) s}\left\{\left(1-e^{-s}\right)^{2} e^{-k^{2}} e^{-s}-1\right\} d s\right] \\
(5.2) & =d_{1} k^{2} e^{k^{2}}\left\{\left(\lambda+k^{2}\right)^{-1}+\int_{0}^{\infty} e^{-\left(\lambda+k^{2}\right) s}\left\{\left(-2 e^{-s}+e^{-2 s}\right)-k e^{-s}\left(1-e^{-s}\right)^{2}+\cdots\right\} d s\right\} \\
& =d_{1} k^{2} e^{k^{2}}\left\{\left(\lambda+k^{2}\right)^{-1}-\frac{\lambda+k^{2}+3}{\left(\lambda+k^{2}+1\right)\left(\lambda+k^{2}+2\right)}\right. \\
& \left.-\frac{2 k^{2}}{\left(\lambda+k^{2}+1\right)\left(\lambda+k^{2}+2\right)\left(\lambda+k^{2}+3\right)}+\cdots\right\}
\end{aligned}
$$


in which the expansion is valid for small $k$. Solving $\tilde{\Psi}(\lambda, k)=1$ results in the dispersion relation

$$
\lambda=\left(d_{1}-1\right) k^{2}-d_{1}\left(\frac{3}{2} d_{1}-1\right) k^{4}+O\left(k^{6}\right) .
$$

According to (2.15), $\theta^{\prime}>0$. We shall discuss the function $\delta(\phi)$ in $\S 9$ and argue that $\delta^{\prime}<0$. Therefore from $(2.8)$ and $(4.33)$ we have

$$
d_{1}=-\frac{\sigma^{\prime}}{\sigma} \phi_{0}=\left(\frac{\theta^{\prime}}{\theta}-\frac{\delta^{\prime}}{\delta}\right) \phi_{0}>0
$$

If $d_{1}<1$, then $\lambda<0$ and the problem is stable for small $k$. In fact further analysis shows that in this case it is stable for all $k$. If $d_{1}>1$, i.e.

$$
1+\frac{\sigma^{\prime}}{\sigma} \phi_{0}<0
$$

then $\lambda>0$ and the problem is unstable for some interval of small $k$. It is restabilized for somewhat larger $k$ by the negative $k^{4}$ term. In this case the maximally unstable wavenumber is

$$
\bar{k}=\left(\frac{d_{1}-1}{3 d_{1}^{2}-2 d_{1}}\right)^{1 / 2} .
$$

This expression, which comes from the small $k$ expansion for $\lambda$, is valid only if $d_{2}-1$ is small.

Finally we translate this dispersion relation to a relation for $\nu$ and $l$, the frequency and wavenumber corresponding to the original variables $x$ and $t$ before the transformation (4.9). The resulting dispersion relation is

$$
\nu=\frac{\sigma}{c \theta}\left(d_{1}-1\right) l^{2}-\frac{\sigma^{2}}{c^{3} \theta^{3}} d_{1}\left(\frac{3}{2} d_{1}-1\right) l^{4}+O\left(l^{6}\right),
$$

in which $d_{1}=-\sigma^{\prime} \phi_{0} / \sigma$.

6. The nonlinear theory. If the spatially homogeneous solution is linearly unstable for some band of wavenumbers, then nonlinear effects will become important. Using a moment method we shall develop a description for the nonlinear evolution of the distribution. For simplicity we shall consider spatial variations only in the vertical direction with vertical spatial component denoted by $x$ and with corresponding velocity component $\xi_{1}$.

Because the spatial variations are one-dimensional, the incompressibility condition $(2.25) \nabla \cdot u=0$ becomes $u=$ constant. Therefore we may take

$$
u=0,
$$

so the scaled Fokker-Planck equation (3.15) is

$$
\varepsilon f_{t}+\xi_{1} \nabla f+\nabla_{\xi} \cdot\left[\left(\alpha \gamma \hat{z}-\varepsilon^{-1} c \theta \xi\right) f\right]=\varepsilon^{-1} c \delta \nabla_{\xi}^{2} f,
$$

in which $\nabla=\partial / \partial x$.

Define moments

$$
m^{(k)}(x, t)=\int \xi_{1}^{k} f(\xi, x, t) d \xi
$$


with $m^{(0)}=\phi, m^{(1)}=\phi v$. Take moments of (6.2) to obtain

$$
\varepsilon \phi_{t}+\nabla m^{(1)}=0
$$

$$
\varepsilon m_{t}^{(1)}+\nabla m^{(2)}-\left(\alpha \gamma \phi-\varepsilon^{-1} c \theta m^{(1)}\right)=0 \text {, }
$$

$$
\varepsilon m_{t}^{(k)}+\nabla m^{(k+1)}-k\left(\alpha \gamma m^{(k-1)}-\varepsilon^{-1} c \theta m^{(k)}\right)=\varepsilon^{-1} c \delta k(k-1) m^{(k-2)},
$$

for $k \geqq 2$. Denote

$$
\beta=\frac{1}{c \theta}, \quad \bar{v}=\frac{\alpha \gamma}{c \theta}, \quad \sigma=\frac{\delta}{\theta},
$$

and rearrange (6.4)-(6.6) as

$$
\begin{aligned}
& \phi_{t}+\frac{1}{\varepsilon} \nabla m^{(1)}=0, \\
& m^{(1)}=\varepsilon \bar{v} \phi-\varepsilon \beta \nabla m^{(2)}-\varepsilon^{2} \beta m_{t}^{(1)}, \\
& m^{(k)}=\sigma(k-1) m^{(k-2)}+\varepsilon \bar{v} m^{(k-1)}-\varepsilon \beta k^{-1} \nabla m^{(k+1)}-\varepsilon^{2} \beta k^{-1} m_{t}^{(k)} .
\end{aligned}
$$

These are solved asymptotically for $\varepsilon$ small by expanding

$$
m^{(k)}=m_{0}^{(k)}+\varepsilon m_{1}^{(k)}+\cdots \text {. }
$$

However to avoid expanding $\beta, \bar{v}$, and $\sigma$, we shall not expand $\phi$. As a result $\phi$ and $m_{i}^{(k)}$ will be dependent on $\varepsilon$. In the resulting equations for $m_{i}^{(k)}$, we find that

$$
\begin{array}{ll}
m_{i}^{(0)}=0 & \text { for } i \neq 0 \quad(\phi \text { is unexpanded }), \\
m_{i}^{(k)}=0 & \text { if } k+i \text { is odd. }
\end{array}
$$

The remaining equations for $m_{i}^{(k)}$ are

$$
\begin{aligned}
\phi_{t} & +\nabla\left(m_{1}^{(1)}+\varepsilon^{2} m_{3}^{(1)}\right)=0, \\
m_{1}^{(1)} & =\bar{v} \phi-\beta \nabla m_{0}^{(2)}, \\
m_{3}^{(1)} & =-\beta \nabla m_{2}^{(2)}-\beta m_{1 t}^{(1)}, \\
m_{0}^{(2)} & =\sigma \phi, \\
m_{2}^{(2)} & =\bar{v} m_{1}^{(1)}-\frac{1}{2} \beta \nabla m_{1}^{(3)}-\frac{1}{2} \beta m_{0 t}^{(2)}, \\
m_{1}^{(3)} & =2 \sigma m_{1}^{(1)}+\bar{v} m_{0}^{(2)}-\frac{1}{3} \beta \nabla m_{0}^{(4)}, \\
m_{0}^{(4)} & =3 \sigma m_{0}^{(2)} .
\end{aligned}
$$

It follows that

$$
\begin{aligned}
m_{1}^{(1)}= & \bar{v} \phi-\beta \nabla \sigma \phi, \\
m_{3}^{(1)}=-\beta(\bar{v} \phi)_{t}+\beta(\beta \nabla(\sigma \phi))_{t} & -\beta \nabla\left\{\bar{v}^{2} \phi-\beta \bar{v} \nabla(\sigma \phi)-\frac{1}{2} \beta(\sigma \phi)_{t}\right. \\
& \left.\quad-\frac{1}{2} \beta \nabla\left(3 \sigma \bar{v} \phi-2 \beta \sigma \nabla(\sigma \phi)-\beta \nabla\left(\sigma^{2} \phi\right)\right)\right\} .
\end{aligned}
$$

Used in (6.14), this gives an evolution equation for the particle density $\phi$.

$$
\text { If } O\left(\varepsilon^{2}\right) \text { terms are ignored the equation for } \phi \text { is }
$$

$$
\phi_{t}+\nabla(\bar{v} \phi-\beta \nabla(\sigma \phi))=0 \text {. }
$$


When linearized around the constant solution $\phi_{0}$, this equation becomes

$$
\phi_{t}+\left(1-d_{2}\right) \bar{v} \nabla \phi=\frac{\sigma}{c \theta}\left(1-d_{1}\right) \nabla^{2} \phi
$$

in which

$$
d_{1}=-\frac{\sigma^{\prime}}{\sigma} \phi_{0}, \quad d_{2}=\left(\frac{\theta^{\prime}}{\theta}-\frac{\gamma^{\prime}}{\gamma}\right) \phi_{0}
$$

and $\sigma, \theta, \gamma$, and $\bar{v}=\alpha \gamma / c \theta$ are evaluated at $\phi_{0}$. If we omit gravity $(\bar{v}=0)$, this diffusion equation has the same dispersion relation as (5.3), to order $k^{2}$.

Next we include $O\left(\varepsilon^{2}\right)$ corrections, which are important if the diffusion coefficient is negative. As a simplification we suppose that the variation in $\phi$ of the drag coefficient $\theta$ and the gravitational coefficient $\gamma$ is smaller than that of the diffusion coefficient. Thus we treat $\beta$ and $\bar{v}$ as constant. The following equation for $\phi$ is found by substituting (6.16) into (6.14):

$$
\begin{array}{r}
\phi_{t}+\bar{v} \nabla \phi-\beta \nabla^{2}(\sigma \phi)+\varepsilon^{2}\left\{-\beta \bar{v} \nabla \phi_{t}-\beta \bar{v}^{2} \nabla^{2} \phi+\frac{3}{2} \beta^{2} \nabla^{2}(\sigma \phi)_{t}+\frac{5}{2} \beta^{2} \bar{v} \nabla^{3}(\sigma \phi)\right. \\
\left.-\beta^{3} \nabla^{3}(\sigma \nabla(\sigma \phi))-\frac{1}{2} \beta^{3} \nabla^{4}\left(\sigma^{2} \phi\right)\right\}=0 .
\end{array}
$$

The equation further simplifies if we look for a steady traveling wave solution satisfying $\phi_{t}+\bar{v} \nabla \phi=0$. The resulting equation for $\phi$ is

$$
\nabla^{2} \sigma \phi-\varepsilon^{2} \beta^{2} \nabla^{3}\left\{\frac{\bar{v}}{\beta} \sigma \phi-\frac{1}{2} \nabla\left(\sigma^{2} \phi\right)-\sigma \nabla(\sigma \phi)\right\}=0 .
$$

In terms of $A, B, C$ scaling (3.13) the parameters $\beta$ and $\bar{v}$ take the-form

$$
\beta=\frac{B^{2}}{C \theta}, \quad \bar{v}=\frac{A B \gamma}{C \theta},
$$

while $\sigma=\delta / \theta$ does not change and is of order one. We see therefore that for Siano's experiment

$$
\beta=\frac{.5 \times 10^{-3}}{\theta}, \quad \bar{v}=.3\left(\frac{\gamma}{\theta}\right), \quad \text { while } \varepsilon^{2}=3.3 \times 10^{-10} .
$$

A linearized analysis of (6.20) about a constant state (with $\bar{v}=0$ ) has a dispersion relation which agrees with (5.3). In fact this is true without the approximation $\beta=$ constant, used in (6.20), for simplicity.

7. Nonlinear equilibrium. Steady nonlinear solutions are described by equation (6.21) for the particle volume fraction $\phi$. We also omit gravitational effects by setting $\bar{v}=0$. Replace $\varepsilon \beta$ by $\varepsilon$ and integrate (6.21) twice to obtain the equation

$$
\sigma \phi+\varepsilon^{2} h_{1}(\phi) \nabla^{2} \phi+\varepsilon^{2} h_{2}(\phi) \nabla \phi^{2}=a+b x,
$$

in which $a$ and $b$ are constants and

$$
\begin{aligned}
& h_{1}(\phi)=\frac{3}{2} \sigma^{2}+2 \sigma \sigma^{\prime} \phi, \\
& h_{2}(\phi)=5 \sigma \sigma^{\prime}+2 \sigma^{\prime 2} \phi+2 \sigma \sigma^{\prime \prime} \phi .
\end{aligned}
$$

If $a$ and $b$ are of moderate size while $\varepsilon$ is small, then to leading order $c=a+b x$ can be treated as constant when solving (7.1). The resulting (quasi) autonomous 
equation is

$$
\sigma \phi+\varepsilon^{2} h_{1}(\phi) \nabla^{2} \phi+\varepsilon^{2} h_{2}(\phi) \nabla \phi^{2}=c .
$$

It has a (quasi) stationary point $\phi=\phi_{0}$ with

$$
\sigma\left(\phi_{0}\right) \phi_{0}=0 \text {. }
$$

For the linearized flow around $\phi=\phi_{0}, \phi_{0}$ is a saddle point if

$$
h_{1}\left(\phi_{0}\right)\left(\sigma\left(\phi_{0}\right)+\sigma^{\prime}\left(\phi_{0}\right) \phi_{0}\right)<0
$$

and a center point if

$$
h_{1}\left(\phi_{0}\right)\left(\sigma\left(\phi_{0}\right)+\sigma^{\prime}\left(\phi_{0}\right) \phi_{0}\right)>0 .
$$

Since (7.3) is even in $\nabla \phi$, the phase plane is symmetric about $\nabla \phi=0$. It follows that the nonlinear solution of (7.3) near $\phi=\phi_{0}$ is hyperbolic if (7.5) holds and periodic if (7.6) holds. The dynamic instability criterion of $\S 5$ is $\sigma+\sigma^{\prime} \phi<0$. For values of $\phi_{0}$ with $\sigma+\sigma^{\prime} \phi_{0}$ small, $h_{1}\left(\phi_{0}\right)<0$. So $\phi_{0}$ is a saddle point if it is linearly, dynamically stable and a center point if it is linearly, dynamically unstable.

Suppose that $\sigma \phi$ has a single dip in it, as in Fig. 7.1. As $x$ changes the (quasi) stationary point $\phi_{0}$, satisfying

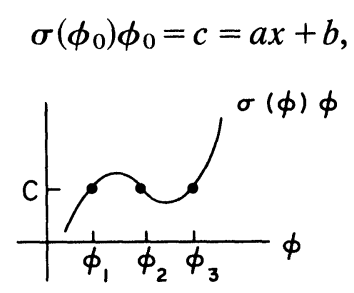

FIG. 7.1. $\sigma \phi$.

changes slowly with $x$. In some range of values of $c$, there are three solutions $\phi_{1}, \phi_{2}$, and $\phi_{3}$ of (7.7) satisfying

$$
\begin{aligned}
& \sigma\left(\phi_{1}\right)+\sigma^{\prime}\left(\phi_{1}\right) \phi_{1}>0, \\
& \sigma\left(\phi_{2}\right)+\sigma^{\prime}\left(\phi_{2}\right) \phi_{2}<0, \\
& \sigma\left(\phi_{3}\right)+\sigma^{\prime}\left(\phi_{3}\right) \phi_{3}>0 .
\end{aligned}
$$

Therefore $\phi_{1}$ and $\phi_{3}$ are saddle points; $\phi_{2}$ is a center point. The two saddle points $\phi_{1}$ and $\phi_{3}$ are connected as in Fig. 7.2b for some special value of $c=\bar{c}$, satisfying

$$
I(\bar{c})=\int_{\phi_{1}}^{\phi_{3}}\{\sigma(\phi) \phi-\bar{c}\} h_{1}(\phi)^{-1} \exp \left\{2 \int^{\phi} \frac{h_{2}\left(\phi_{1}\right)}{h_{1}\left(\phi_{1}\right)} d \phi_{1}\right\} d \phi=0 .
$$

There is only one such value of $\bar{c}$ since $d I / d \bar{c}<0$. If $I \neq 0$, the saddle points are not connected and the phase plane has the form drawn in Fig. 7.2a or $c$.

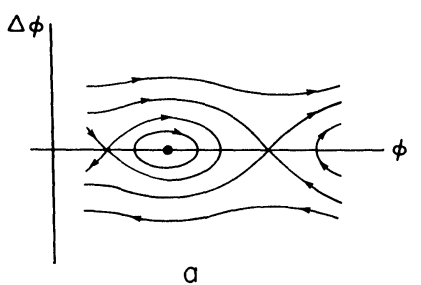

a

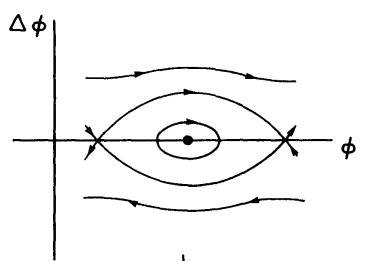

b

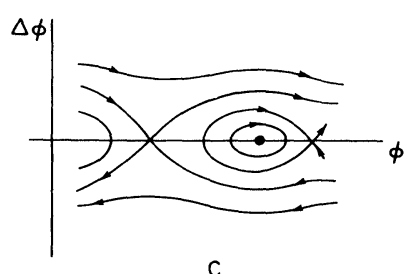

C

FIG. 7.2. Possible phase planes. 
The variation of $\phi$ with $x$ goes approximately as follows. For $a x+b<\bar{c}, \phi(x)=\phi_{1}$, the smallest value of $\phi$ solving $\sigma\left(\phi_{1}\right) \phi_{1}=a x+b$. At $a x+b=\bar{c}$, the solution $\phi$ makes a rapid transition from $\phi_{1}(\bar{c})$ to $\phi_{3}(\bar{c})$ approximately following the flow line connecting these two saddles. For $a x+b>\bar{c}, \phi(x)=\phi_{3}$, the largest value of $\phi$ solving $\sigma\left(\phi_{3}\right) \phi_{3}=$ $a x+b$. Therefore $\phi$ varies slowly in $x$, except near $a x+b=\bar{c}$ where it varies at a rate $1 / \varepsilon$. The resulting profile of $\phi$ is pictured in Fig. 7.3.

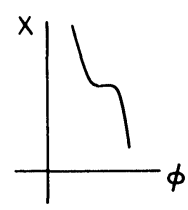

FIG. 7.3. Spatial variation of $\phi$.

8. The $N$-particle Kramers-Smoluchovski approximation. In this and the next section we shall consider some problems of a more general character regarding interacting particle systems. We shall not derive the nonlinear Fokker-Planck equation (2.11) from first principles. However, we shall discuss the derivation of a nonlinear diffusion equation from an infinite particle system. Before doing this we shall describe the (linear) Kramers-Smoluchovski limit [20].

Let $x^{(1)}, x^{(2)}, \cdots, x^{(N)}$ and $\xi^{(1)}, \xi^{(2)}, \cdots, \xi^{(N)}$ denote the positions and velocities of $N$-particles in $\mathbb{R}^{3}$ having equal mass $M$, interacting with each other via the fluid in which they are immersed and being acted upon by Brownian forces. Let $u(x), p(x)$ denote the fluid velocity and pressure respectively. The equations of motion are

$$
\begin{aligned}
& \frac{d x^{(j)}}{d t}=\xi^{(j)}, \\
& M \frac{d \xi^{(j)}}{d t}=-F^{(j)}\left(x^{(1)}, \cdots, x^{(N)}, \xi^{(1)}, \cdots, \xi^{(N)}\right)+\sigma \frac{d w^{(j)}}{d t}, \\
& \mu \Delta u-\nabla p=0, \quad \nabla \cdot u=0, \quad u \rightarrow 0 \quad \text { as } x \rightarrow \infty, \\
& u=\xi^{(j)} \text { on }\left|x-x^{(j)}\right|=a, \\
& F^{(j)}=\int_{\left|x-x^{(j)}\right|=a} \tau \cdot n d S(x), \quad \tau_{l k}=-p \delta_{l k}+\mu\left(u_{l, k}+u_{k, l}\right), \\
& \quad l, k=1,2,3, \quad j=1,2, \cdots, N .
\end{aligned}
$$

Here $n$ denotes the unit outward normal to the surface of the spherical particles. Note that the inertial terms have been dropped in (8.3) because we assume that the fluid adjusts rapidly to changes in the configuration of the particles. Because of this assumption we may regard $u$ as a function of $x^{(1)}, \cdots, x^{(N)}, \xi^{(1)}, \cdots, \xi^{(N)}$

$$
u=u\left(x ; x^{(1)}, \cdots, x^{(N)}, \xi^{(1)}, \cdots, \xi^{(N)}\right) .
$$

In fact $u$ is a linear function of the $\xi^{(j)}$ and, in view of $(8.5), F^{(j)}$ in (8.2) is indeed a function of $x^{(1)}, \cdots, x^{(N)}$ and a linear function of $\xi^{(1)}, \cdots, \xi^{(N)}$. We may write

$$
F^{(j)}=\sum_{l=1}^{N} A^{j l} \xi^{(l)}, \quad j=1,2, \cdots, N
$$

where for each $j$ and $l, A^{j l}$ is a $3 \times 3$ matrix $\left(A_{i k}^{j l}\right)$, the resistance matrix. These matrices 
are functions of $x^{(1)}, \cdots, x^{(N)}$, are symmetric [14]

$$
A_{i k}^{j l}=A_{k i}^{l j}
$$

and they are positive definite. In $(8.2)$ the $w^{(j)}(t), j=1, \cdots, N$ are independent Brownian motion processes and $\sigma=\sigma\left(x^{(1)}, x^{(2)}, \cdots, x^{(N)}\right)$ is the noise level defined to conform with equilibrium thermodynamics. The Fokker-Planck equation for the $N$-particle density $f^{(N)}\left(t, x^{(1)}, \cdots, x^{(N)}, \xi^{(1)}, \cdots, \xi^{(N)}\right)$ takes the form

$$
\frac{\partial f^{(N)}}{\partial t}+\sum_{j=1}^{N} \xi^{(j)} \cdot \frac{\partial f^{(N)}}{\partial x^{(j)}}+\sum_{j=1}^{N} \frac{\partial}{\partial \xi^{(j)}} \cdot\left[-\frac{F^{(j)}}{M} f^{(N)}-\frac{\sigma^{2}}{2 M^{2}} \frac{\partial f^{(N)}}{\partial \xi^{(j)}}\right]=0 .
$$

The Smoluchovski-Kramers limit amounts to a small mean free time limit for this $N$-particle process. The "collision" term, the third term on the left in (8.9), is assumed to be large compared to the other two terms. We can then carry out a fluid-limit calculation based on the equilibrium solution

$$
f_{0}^{(N)}=\phi_{0} \frac{e^{-\Phi(\xi, \xi) / \sigma^{2}}}{z}
$$

where

$$
\begin{aligned}
& \Phi(\xi, \xi)=M \sum \xi^{(j)} \cdot A^{j l}\left(x^{(1)}, \cdots, x^{(N)}\right) \xi^{(l)}, \\
& z=\int \cdots \int e^{-\Phi(\xi, \xi) / \sigma^{2}} d \xi^{(1)}, \cdots, d \xi^{(N)},
\end{aligned}
$$

and $\phi_{0}$ is a positive function of $x$ and $t$. The function $f_{0}$ satisfies the equilibrium equation

$$
\sum_{j=1}^{N} \frac{\partial}{\partial \xi^{(j)}} \cdot\left[-\frac{F^{(j)}}{M} f_{0}-\frac{\sigma^{2}}{\partial M^{2}} \frac{\partial f_{0}}{2 \xi^{(j)}}\right]=0 .
$$

By an asymptotic analysis that is very similar to the one used in $\S 6$ one arrives at a diffusion equation for the function $\phi_{0}\left(t, x^{(1)}, \cdots, x^{(N)}\right)$ which has the form

$$
\frac{\partial \phi_{0}}{\partial t}=\sum_{j=1}^{\cdot N} \frac{\partial}{\partial x^{(j)}} \cdot\left[\bar{D}^{j l}\left(x^{(1)}, \cdots, x^{(N)}\right) \frac{\partial \phi_{0}}{\partial x^{(l)}}+\bar{E}^{j}\left(x^{(1)}, \cdots, x^{(N)}\right) \phi_{0}\right] .
$$

The determination of $\bar{D}^{j l}$ and $\bar{E}^{j}$ is complicated and we shall omit it (a similar computation for a simpler case is given in [23]). We remark only that the diffusion tensor $\bar{D}^{j l}$ is proportional to the reciprocal of the tensor $A^{j l}$ squared. So its dependence on $x^{(1)}, x^{(2)}, \cdots, x^{(N)}$ is quite involved.

The interacting Brownian motion model of the next section is an infinite $(N=\infty)$ particle version of (8.13), much simplified to allow focusing on collective effects.

9. Effective diffusivity of a Brownian particle in a swarm of other particles. The nonlinear Fokker-Planck equation (2.11), which is the starting point for our analysis, can itself be derived as an asymptotic limit of $N$ interacting stochastic processes as $N \rightarrow \infty$ in a manner analogous to propagation of chaos results [18] but for spatially inhomogeneous systems. Our calculations regarding this are at present incomplete and we cannot argue directly that the velocity diffusion coefficient $D \delta(\phi)$ is a decreasing function of the spatial concentration $\phi$. We approach this issue via another problem which we now describe.

Let $\Phi(x)$ be a smooth positive function of compact support that is even, $\Phi(x)=$ $\Phi(-x), x \in \mathbb{R}^{3}$. We consider an infinite system of Brownian motions that interact with each other with pair potential $\Phi(x)$. This means that if $\left(x^{(1)}, x^{(2)}, \cdots\right)$ are the coordi- 
nates of these particles and if $\nabla_{j}$ is the gradient with respect to the coordinates of the jth particle then

$$
\mathscr{L}=\sum_{j=1}^{\infty} \nabla_{j}^{2}+\sum_{j=1}^{\infty} \sum_{k \neq j}\left(\nabla_{j} \Phi\left(x_{j}-x_{k}\right)\right) \nabla_{j}
$$

is the infinitesimal generator of the process or, what amounts to the same thing, the formal joint probability density of these particles satisfies

$$
\frac{\partial P}{\partial t}=\mathscr{L}^{*} P, \quad P=P\left(t, x^{(1)}, x^{(2)}, \cdots\right),
$$

which is the Fokker-Planck equation. We shall not give a full mathematical treatment here so we do not go into the detailed construction of this infinite particle process [6]. Let $P_{\mu}$ denote the Poisson distribution of $\mathbb{R}^{3}$ with intensity $\mu$. Thus, the distribution of the particles in a set $A \subset \mathbb{R}^{3}$ is Poisson with parameter $\mu$ times the volume of $A$. One can verify that formally the distributions

$$
G_{\mu}=\exp \left(\frac{1}{2} \sum_{i \neq j} \Phi\left(x_{i}-x_{j}\right)\right) P_{\mu}
$$

are invariant for the evolution (9.2). That is, if $P$ at $t=0$ in (9.2) is given by $G_{\mu}$ then $P=G_{\mu}$ for all time. Again this statement must be made precise since for example the expression (9.3) is not well defined as it stands [6].

With this infinite particle process we can associate effective one-particle diffusion coefficients in two different ways.

(i) The self-diffusion or tagged particle diffusion coefficient.

(ii) The bulk or mean field diffusion coefficient.

The first one is obtained if we focus attention "on one particle $x(t)=x^{(1)}(t)$ say, and show that

$$
(x(t)-x(0))^{2} \sim \tilde{a}(\mu) t, \quad t \uparrow \infty,
$$

where $\tilde{a}(\mu)$ is a constant depending on the concentration $\mu$ of the other particles at $t=0$, distributed according to (9.3). The constant $\tilde{a}(\mu)$ is the self-diffusion coefficient. It is not difficult to see that $\tilde{a}(\mu)$ is necessarily a decreasing function of $\mu$ for repulsive potentials $\Phi$ : the more particles there are about $x(t)=x^{(1)}(t)$ the smaller its effective diffusivity will be since the other particles will tend to obstruct its Brownian motion.

The bulk diffusion coefficient is obtained in a different way. We consider the evolution (9.2) with initial density close to the equilibrium distribution (9.3) but different from it. Then the motion of the system of particles can be represented by a mean field $\phi(t, x)=$ the average density of particles at $x$ at time $t$. The mean field satisfies a nonlinear diffusion equation

$$
\phi_{t}=\nabla \cdot(\hat{a}(\phi) \nabla \phi),
$$

and $\hat{a}(\mu)$ is called the bulk diffusivity. We can think of (9.5) as representing the Fokker-Planck equation of an "effective" particle $\bar{x}(t)$ which undergoes Brownian motion with diffusivity a function of the local concentration.

It is well known that the self-diffusivity and the bulk diffusivity are in general different. In fact, the bulk diffusivity is an increasing function of the concentration here, at low concentrations [4]. Physically this means that at low concentrations the effective particle $\bar{x}(t)$ feels a drift that pushes it into regions of lower concentration faster when the local concentration is higher. 
The Fokker-Planck equation (2.11) is the analogue of (9.5) when the underlying system of interacting particles is not interacting Brownian motions but instead interacting Ornstein-Uhlenbeck processes (i.e. diffusion in velocity space rather than in physical space). The diffusion coefficient $D \delta(\phi)$ is the bulk diffusion coefficient in velocity space. Now however it depends on the spatial concentration and this behavior is more like the one of the self-diffusion coefficient, i.e. $D \delta(\phi)$ decreases with concentration. The "effective" Ornstein-Uhlenbeck particle $(\bar{x}(t), \bar{\xi}(t))$ whose density satisfies (2.11) diffuses in velocity space more sluggishly when there are lots of particles around.

This qualitative discussion is of course no demonstration that $D \delta(\phi)$ decreases with $\phi$ when $\phi$ is small. What we have shown to date is that the self-diffusion coefficient $\tilde{a}(\mu)$ for the system described by (9.1) exists and is a decreasing function of $\mu$ [6]. The existence of $\hat{a}(\phi)$ in (9.5) from an infinite particle model, or $D \delta(\phi)$ in (2.11), has not been shown while properties of $\hat{a}(\phi)$ for $\phi$ small follow from Batchelor's calculations [4].

We conclude by noting that one may apply the propagation of chaos limit (obtain a mean field equation) at the level of interacting Ornstein-Uhlenbeck processes and then proceed with a nonlinear Kramers-Smoluchovski limit (as we did in § 6). Alternatively one can apply a Kramers-Smoluchovski limit at the $N$-particle level $(\S 8)$ and then a mean field limit afterward resulting in (9.5).

10. Conclusions. Through the linear theory of $\S \S 4$ and 5 or the nonlinear theory of $\S \S 6$ and 7 , we have demonstrated a mechanism for linear instability of a homogeneous suspension and nonlinear restabilization. To understand the underlying physics, we examine the effects of velocity diffusion.

A particle undergoes velocity diffusion due to many collisions in each of which it changes its velocity slightly. It thus moves chaotically through velocity space, but smoothly through physical space. In the nonlinear Kramers-Smoluchovski limit (described in $\S 6$ ), the strength of the velocity diffusion is very large (or alternatively the time and space scales are very long). Then the physical path also looks chaotic, and the particle undergoes diffusion in physical space.

Now suppose, as in this problem, that the variance $\sigma=\delta / \theta$ of the velocity diffusion is a decreasing function of the density $\phi$. At points where $\phi$ is large, the velocity diffusion is weak; where it is small, the diffusion is strong. It follows that particles leave regions where $\phi$ is small and get stuck in regions where $\phi$ is large. This is possible because in velocity diffusion particles are not moving down their spatial gradient as they would in spatial diffusion. Therefore if $\phi$ starts as a constant plus a sinusoidal term (as in Fig. 1), on the average particles will move from the troughs of $\phi$ to its peaks. This amplification of the sinusoidal component is the instability described in the preceding sections. Mathematically it is represented by the negative diffusion coefficients in the continuum equations, e.g. in (5.7) and (6.20).

This negative diffusion with a restabilizing fourth order term is the same as the Cahn-Hilliard equation [7] proposed in an ad hoc manner by Siano [25] to explain the instabilities in his experiments. It is also being studied in this context by Cohen and Hagan [9]. Our contribution is to successfully derive this equation from more basic physics. We also show that it has nonlinear equilibrium solutions which contain rapid variations similar to those observed by Siano. However we are unable to explain the entire "staircase" solution that he found.

We note finally that our instability and restabilization results embodied in the Cahn-Hilliard equation (6.20) could not have been obtained by modeling the suspension as an interacting system of Brownian motions. We saw in $\S 9$ that the bulk 

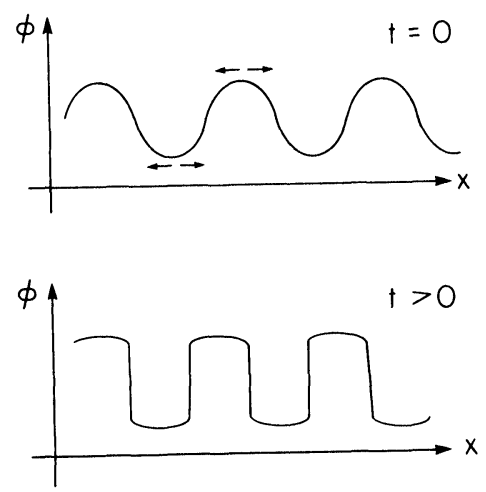

FIG. 1. Note. Curves have same period and same mean value but the amplitude oscillation is steeper for $t>0$.

diffusivity in that case would be an increasing function of the concentration (as was shown by Batchelor [4] for dilute suspension). It is therefore important to formulate the problem in phase space as we do in $\$ 2$. A possible explanation for the decrease of $D \delta(\phi)$ in (2.9) is given in $\S 9$ by analogy with known properties of the self-diffusion coefficient.

Appendix A. Two-fluid models of a suspension. A two-fluid model is a commonly used description of a suspension and is simpler than the Fokker-Planck model described in $\S 2$. We shall perform a linearized stability analysis of the steady solution for such a model. In particular we shall show that when diffusive effects are much stronger than gravitational effects, this model predicts stability for all wavenumbers. Therefore such a simple model cannot describe the instabilities found in $\S \S 4$ and 5 or the approximate nonlinear states found in $\S \S 6$ and 7 .

The two-fluid equations for a suspension [13], [15] are

$$
\begin{aligned}
& \frac{\partial}{\partial t} \phi+\nabla \cdot\left(v_{p} \phi\right)=0, \\
& \frac{\partial}{\partial t}(1-\phi)+\nabla \cdot\left(v_{f}(1-\phi)\right)=0,
\end{aligned}
$$

$$
\phi \rho_{p}\left(\frac{\partial}{\partial t} v_{p}+v_{p} \cdot \nabla v_{p}-\mathbf{g}\right)=-\phi \nabla p-\phi(1-\phi) S\left(v_{p}-v_{f}\right)-\phi \sigma \nabla \phi
$$

$$
(1-\phi) \rho_{f}\left(\frac{\partial}{\partial t} v_{f}+v_{f} \cdot \nabla v_{f}-\mathbf{g}\right)
$$

$$
=-(1-\phi) \nabla p+\phi(1-\phi) S\left(v_{p}-v_{f}\right)+\mu(1-\phi) \nabla^{2} v_{f}
$$

In the model the effect of diffusion of the particles is included though the term $\phi \sigma \nabla \phi$ as in [15]. For algebraic simplicity we have written the diffusion coefficient as $\phi \sigma$, although $\phi \sigma=O(1)$ for small $\phi$. Similarly we have replaced the usual coefficient of Stokes drag by $(1-\phi) S$. The gravitational vector pointing downwards is denoted by $\mathbf{g}$.

Dividing by $\phi$ and $(1-\phi)$ changes the momentum equations to

$$
\rho_{p}\left(\frac{\partial}{\partial t} v_{p}+v_{p} \cdot \nabla v_{p}-\mathbf{g}\right)=-\nabla p-(1-\phi) S\left(v_{p}-v_{f}\right)-\sigma \nabla \phi
$$

$$
\rho_{f}\left(\frac{\partial}{\partial t} v_{f}+v_{f} \cdot \nabla v_{f}-\mathbf{g}\right)=-\nabla p+\phi S\left(v_{p}-v_{f}\right)+\mu \Delta^{2} v_{f}
$$


The system (A.1), (A.2), (A.5), (A.6) has a steady solution with $\phi$ constant and

$$
\begin{aligned}
& p=-\left(\phi \rho_{p}+(1-\phi) \rho_{f}\right) \mathbf{g} \cdot \mathbf{x}, \\
& v_{p}-v_{f}=S^{-1}\left(\rho_{p}-\rho_{f}\right) \mathbf{g} .
\end{aligned}
$$

Denote this solution by $\Phi, P, v_{p}=V$ and set $v_{f}=0$ by Galilean transformation. Then look for a perturbed solution

$$
\Phi+\phi, \quad P+p, \quad V+v_{p}, \quad v_{f},
$$

with $\phi, p, v_{p}$ and $v_{f}$ small and satisfying the linearized equations

$$
\frac{\partial}{\partial t} \phi+V \cdot \nabla \phi+\Phi \nabla \cdot v_{p}=0
$$

$$
-\frac{\partial}{\partial t} \phi+(1-\Phi) \nabla \cdot v_{f}=0
$$

$$
\rho_{p}\left(\frac{\partial}{\partial t} v_{p}+V \cdot \nabla v_{p}\right)=-\nabla p-(1-\Phi) S\left(v_{p}-v_{f}\right)-(1-\Phi) S^{\prime} \phi V+\phi S V-\sigma \nabla \phi
$$

$$
\rho_{f} \frac{\partial}{\partial t} v_{f}=-\nabla p+\Phi S\left(v_{p}-v_{f}\right)+\Phi S^{\prime} \phi V+\phi S V+\mu \nabla^{2} v_{f}
$$

in which $\boldsymbol{S}^{\prime}=d \boldsymbol{S}(\Phi) / d \phi$.

Now look for a solution which varies sinusoidally only in the vertical direction, with vertical coordinate denoted by $x$. Set

$$
\left(\phi, v_{p}, v_{f}, p\right)=\left(\bar{\phi}, \bar{v}_{p}, \bar{v}_{f}, \bar{p}\right) e^{i k x+\omega t}
$$

From (A.10)-(A.13) we find that

$$
(\omega+i k V) \bar{\phi}+i k \Phi \bar{v}_{p}=0 \text {, }
$$

$$
-\omega \bar{\phi}+i k(1-\Phi) \bar{v}_{f}=0 \text {, }
$$

$$
\rho_{1}(\omega+i k V) \bar{v}_{p}=-i k \bar{p}-(1-\Phi) S\left(\bar{v}_{p}-\bar{v}_{f}\right)-(1-\Phi) S^{\prime} V \bar{\phi} j S V \bar{\phi}-i k \sigma \bar{\phi}
$$

Eliminate $\bar{p}$ from (A.17) and (A.18), then use (A.15) and (A.16) to eliminate $\bar{v}_{p}$ and $\bar{v}_{f}$. After division by $\bar{\phi}$ this yields the dispersion relation

$$
A \omega^{2}+B \omega+C=0
$$

with

$$
\begin{aligned}
& A=\rho_{p}(1-\Phi)+\rho_{f} \Phi \\
& B=S+\mu \Phi k^{2}+i 2 \rho_{1} V(1-\Phi) k \\
& C=\left\{\left(\sigma \Phi-\rho_{1} V^{2}\right) k^{2}+i k V\left(S-S^{\prime} \Phi\right)\right\}(1-\Phi)
\end{aligned}
$$

The solutions

$$
\omega_{ \pm}=(2 A)^{-1}\left\{-B \pm \sqrt{B^{2}-4 A C}\right\}
$$

have real parts

$$
\operatorname{Re} \omega_{ \pm}=(2 A)^{-1}\left\{-c \pm\left(\frac{1}{2} a+\frac{1}{2}\left(a^{2}+b^{2}\right)^{1 / 2}\right)^{1 / 2}\right\}
$$


in which

$$
\begin{aligned}
c & =\operatorname{Re} B=S+\mu \Phi k^{2}, \\
a & =c^{2}+a_{1} k^{2}, \\
a_{1} & =4 \Phi(1-\Phi)\left(\rho_{p} \rho_{f} V^{2}-\sigma A\right), \\
b & =b_{1} k+b_{2} k^{3}, \\
b_{1} & =4(1-\Phi) V S\left(\rho_{p}-A\left(1-\Phi S^{\prime} / S\right)\right), \\
b_{2} & =4(1-\Phi) \Phi V \mu \rho_{p} .
\end{aligned}
$$

Since $\operatorname{Re} \omega_{-} \leqq 0$, this mode is always stable.

The stability criterion $\operatorname{Re} \omega_{+} \leqq 0$ can be written as $c \geqq\left(\frac{1}{2} a+\frac{1}{2}\left(a^{2}+b^{2}\right)^{1 / 2}\right)^{1 / 2}$ or

$$
-4\left(S+\mu \Phi k^{2}\right)^{2} a_{1} k^{2} \geqq\left(b_{1}+b_{2} k^{2}\right)^{2} k^{2} .
$$

Since equality can hold for at most one value of $k^{2} \neq 0$, we need only investigate this inequality for $k^{2}$ large and small. The stability criterion for small $k \neq 0$ is $-4 S^{2} a_{1}>b_{1}^{2}$ or

$$
A \sigma \Phi>V^{2}\left\{(1-\Phi)\left(\rho_{p}-A\left(1-\Phi S^{\prime} / S\right)\right)+\rho_{p} \rho_{f} \Phi\right\}
$$

in which $A=\rho_{p}(1-\Phi)+\rho_{f} \Phi$ as in (A.20). The stability criterion for large $k$ is $-4 \mu^{2} \Phi^{2} a_{1}>b_{2}^{2}$ or

$$
A \sigma \Phi>V^{2}\left\{\rho_{p}^{2}(1-\Phi)+\rho_{p} \rho_{f} \Phi\right\}
$$

From (A.24) and (A.25) we see that the instability is driven by gravity, which forces $V=S^{-1}\left(\rho_{p}-\rho_{f}\right) g \neq 0$. If there is no diffusion $(\sigma=0)$, the steady solution is unstable to all wavenumbers $k$. We note though that if $\mu \neq 0, \operatorname{Re} \omega_{+}$has a finite limit as $|k| \rightarrow \infty$, for all $\sigma$.

In the regime considered in this paper, diffusive effects are large compared to gravitational effects. Recall also that $\sigma=O(1 / \Phi)$. Therefore (A.24) and (A.25) are always satisfied and the steady solution is stable.

Acknowledgment. Finally, we want to thank Bud Homsy for his valuable suggestions and encouragement.

\section{REFERENCES}

[1] E. BARneA AND J. MizRAhI, A generalized approach to fluid dynamics of particulate systems I, Chem. Eng. J., 5 (1973), pp. 171-189.

[2] G. K. BAtchelor, Sedimentation in a dilute suspension of spheres, J. Fluid Mech., 52 (1972), pp. 245-268.

[3] - Transport properties of two-phase materials with random structure, Ann. Rev. Fluid Mech., 6 (1974), pp. 227-255.

[4] - Brownian diffusion of particles with hydrodynamic interaction, J. Fluid Mech., 74 (1976), pp. 1-29.

[5] B. J. Berne ANd R. Pecora, Dynamic Light Scattering, John Wiley, New York, 1976.

[6] R. CAflisCh AND G. C. PAPANICOlAOU, Selfdiffusion for interacting Brownian motions, to appear.

[7] J. W. CAHN AND J. E. HilliARD, Free energy of a nonuniform system III. Nucleation in a twocomponent incompressible fluid, J. Chem. Phys., 31 (1959), pp. 688-701.

[8] C. Cercignani, Theory and Applications of the Boltzmann Equation, Elsevier, New York, 1975.

[9] D. COHEN AND P. HAGAN, private communication. 
[10] J. M. CROWLEY, Viscosity-induced instability of a one-dimensional lattice of falling spheres, J. Fluid Mech., 45 (1971), pp. 151-159.

[11] - Clumping instability of a falling horizontal lattice, Phys. Fluids, 19 (1976), pp. 1296-1300.

[12] _ Clumping instability which is not predicted by the nearest neighbor approximation, Phys. Fluids, 20 (1977), p. 339.

[13] D. Drew, Stability of a Stokes layer of a dusty gas, Phys. Fluids, 22 (1979), p. 2081.

[14] J. HAPPEl AND H. BRENNER, Low Reynolds Number Hydrodynamics with Special Applications to Particulate Media, Prentice Hall, Englewood Cliffs, NJ, 1965.

[15] C. D. Hill, Two-dimensional analysis of the stability of particulate sedimentation, Phys. Fluids, 23 (1980), pp. 667-668.

[16] E. J. HINCH, An averaged equation approach to particle interactions in a fluid suspension, J. Fluid Mech., 83 (1977), pp. 695-720.

[17] G. HoMsy, Model equations for wavy viscous film flow, Lectures in Applied Mathematics, 15, American Mathematical Society, Providence, RI, 1974.

[18] M. KAC, Foundations of kinetic theory, Proc. Third Berkeley Symp. on Math. Stat. and Prob., Vol. 3, pp. 171-197.

[19] J. B. Keller, L. A. Rubenfeld AND J. E. Molyneux, Extremum principles for slow viscous flow with applications to suspensions, J. Fluid Mech., 30 (1967), pp. 97-125.

[20] H. Kramers, Brownian motion in a field of force and the diffusion model of chemical reactions, Physica, 7 (1940), pp. 284-304.

[21] A. NOviC-COHEN AND L. A. SEGEL, Nonlinear aspects of the Cahn-Hilliard equation, submitted to Physica D.

[22] D. R. Oliver, The sedimentation of suspensions of closely-sized spherical particles, Chem. Eng. Sci., 15 (1961), pp. 230-242.

[23] G. C. PAPANICOLAOU, Introduction to the asymptotic analysis of stochastic equations, AMS Book Series Vol. 16, Lectures in Applied Mathematics, American Mathematical Society, Providence, RI, 1977, pp. 109-148.

[24] W. B. RuSSEl, Brownian motion of small particles suspended in liquids, Ann. Rev. Fluid Mech., 13 (1981), pp. 425-455.

[25] D. B. SiANO, Layered sedimentation in suspensions of monodisperse spherical colloidal particles, J. Colloid. Interface Sci., 68 (1979), pp. 111-127. 